ARTICLE

Clinical Study

\title{
Phase I studies of AZD1208, a proviral integration Moloney virus kinase inhibitor in solid and haematological cancers
}

\author{
Jorge Cortes ${ }^{1}$, Kenji Tamura ${ }^{2}$, Daniel J. DeAngelo ${ }^{3}$, Johann de Bono ${ }^{4}$, David Lorente ${ }^{4}$, Mark Minden ${ }^{5}$, Geoffrey L. Uy ${ }^{6}$, \\ Hagop Kantarjian ${ }^{1}$, Lisa S. Chen ${ }^{7}$, Varsha Gandhi ${ }^{7}$, Robert Godin ${ }^{8}$, Karen Keating ${ }^{9}$, Kristen McEachern ${ }^{9}$, Karthick Vishwanathan $^{8}$, \\ Janet Elizabeth Pease ${ }^{10}$ and Emma Dean ${ }^{11}$
}

BACKGROUND: Proviral integration Moloney virus (PIM) kinases (PIM1, 2 and 3) are overexpressed in several tumour types and contribute to oncogenesis. AZD1208 is a potent ATP-competitive PIM kinase inhibitor investigated in patients with recurrent or refractory acute myeloid leukaemia (AML) or advanced solid tumours.

METHODS: Two dose-escalation studies were performed to evaluate the safety and tolerability, and to define the maximum tolerated dose (MTD), of AZD1208 in AML and solid tumours. Secondary objectives were to evaluate the pharmacokinetics, pharmacodynamics (PD) and preliminary efficacy of AZD1208.

RESULTS: Sixty-seven patients received treatment: 32 in the AML study over a 120-900 mg dose range, and 25 in the solid tumour study over a $120-800 \mathrm{mg}$ dose range. Nearly all patients (98.5\%) in both studies experienced adverse events, mostly gastrointestinal (92.5\%). Dose-limiting toxicities included rash, fatigue and vomiting. AZD1208 was not tolerated at $900 \mathrm{mg}$, and the protocoldefined MTD was not confirmed. AZD1208 increased CYP3A4 activity after multiple dosing, resulting in increased drug clearance. There were no clinical responses; PD analysis showed biological activity of AZD1208.

CONCLUSIONS: Despite the lack of single-agent clinical efficacy with AZD1208, PIM kinase inhibition may hold potential as an anticancer treatment, perhaps in combination with other agents.

British Journal of Cancer (2018) 118:1425-1433; https://doi.org/10.1038/s41416-018-0082-1

\section{INTRODUCTION}

Proviral integration Moloney virus (PIM) kinases (PIM1, 2 and 3) are a family of nuclear and cytoplasmic serine $(\mathrm{S}) /$ threonine $(\mathrm{T})$ kinases that regulate apoptosis and control cell cycle progression by phosphorylating substrates such as $\mathrm{Bcl}-2$ antagonist of cell death (BAD), ${ }^{1,2}$ p21Cip1/WAF1 ${ }^{3}$ and $\mathrm{cMyb}^{4}$

PIM kinases appear overexpressed in several tumour types, contributing to oncogenesis., ${ }^{5,6}$ For example, PIM1 is overexpressed in $\sim 30 \%$ of haematopoietic malignancies, particularly in acute myeloid leukaemia (AML) and acute lymphoblastic leukaemia. ${ }^{7}$ PIM1 in T cells induces the formation of lymphoma and increases the rate of lymphoma development in response to Murine Leukaemia Virus. ${ }^{6}$ Furthermore, PIM1 and PIM2 are overexpressed in haematological malignancies ${ }^{8,9}$ and solid tumours. 9,10

AZD1208 is a potent, ATP-competitive, pan-PIM kinase inhibitor designed to target PIM1, 2 and $3 .^{11}$ It has been investigated in preclinical models of $A M L$ and prostate cancer. ${ }^{12,13}$ In AML cell lines, inhibition of cell growth by AZD1208 correlated with PIM1 expression. AZD1208 induced cell cycle arrest and apoptosis, which were accompanied by dose-dependent reductions in levels of phosphorylated BAD, 4E-BP1, p70S6K and S6 proteins. ${ }^{12}$

Here, we report the results of two parallel phase I doseescalation studies using the PIM kinase inhibitor, AZD1208, which examined the safety, tolerability, pharmacokinetics (PK) and preliminary efficacy of AZD1208 in patients with recurrent or refractory AML or advanced solid tumours.

\section{MATERIALS AND METHODS}

Study objectives

Both dose-escalation studies recruiting patients with AML (ClinicalTrials.gov, NCT01489722) and advanced solid malignancies (NCT01588548) were phase I, open-label, multicentre studies designed to identify the maximum tolerated dose (MTD) and evaluate the safety and tolerability of AZD1208 administered

\footnotetext{
'Department of Leukemia, Division of Cancer Medicine, The University of Texas MD Anderson Cancer Center, 1901 East Road, Houston, TX 77054, USA; ${ }^{2}$ Department of Breast Oncology and Medical Oncology, National Cancer Center Hospital, 5-1-1 Tsukiji, Chuo, Tokyo 104-0045, Japan; ${ }^{3}$ Department of Medical Oncology, Dana-Farber Cancer Institute, 450 Brookline Avenue, Room D-2050, Boston, MA 02215, USA; ${ }^{4}$ Prostate Cancer Targeted Therapy Group and Drug Development Unit, Royal Marsden, Downs Road, Sutton, Surrey SM2 5PT, UK; ${ }^{5}$ Division of Stem Cell and Developmental Biology, Ontario Cancer Institute, Princess Margaret Hospital, 610 University Avenue, Toronto, ON M5G 2M9, Canada; ${ }^{6}$ Department of Medicine, Oncology Division, Washington University School of Medicine, 660 S. Euclid Ave., St. Louis, MO 63110, USA; ${ }^{7}$ Department of Experimental Therapeutics, Division of Cancer Medicine, The University of Texas MD Anderson Cancer Center, 1901 East Road, Houston, TX 77054, USA; ${ }^{8}$ AstraZeneca, 35 Gatehouse Dr, Waltham, MA 02451,

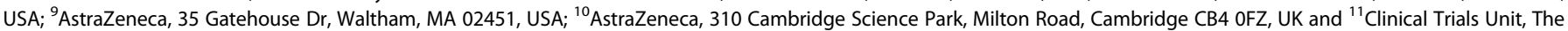
Christie NHS Foundation Trust, Manchester M20 4BX, UK

Correspondence: Emma Dean (emma.dean@astrazeneca.com)
}

Received: 26 October 2017 Revised: 22 March 2018 Accepted: 22 March 2018

Published online: 16 May 2018 
orally once daily (QD). Secondary objectives included evaluation of the drug PK and preliminary evidence of efficacy. The AML study also explored pharmacodynamic (PD) biomarkers.

Patient eligibility

Prior to participation in either study, all patients signed an informed consent document approved by the Institutional Review Board at each site. Both studies were conducted according to the Declaration of Helsinki.

For the AML study, eligible patients were $\geq 18$ years of age with relapsed/refractory $A M L$, with $A M L$ secondary to myelodysplastic syndromes or myeloproliferative neoplasm, or with chronic myeloid leukaemia in blast phase. Patients were required to have Eastern Oncology Cooperative Group Performance Status (ECOG PS) $0-2$ and be considered likely to complete at least 4 weeks of therapy.

For the solid tumour study, eligible patients were $\geq 18$ years of age and diagnosed with advanced solid tumours-including nonHodgkin lymphoma-refractory to standard therapies, or for which no standard therapies exist. Eligible patients were required to have ECOG PS $0-1$, life expectancy of $\geq 12$ weeks, and $\geq 1$ lesion that could be accurately assessed by Response Evaluation Criteria in Solid Tumours (RECIST) v.1.1 using computed tomography (CT) or magnetic resonance imaging.

Patients were excluded from both studies if they had concomitant uncontrolled diseases, including uncontrolled diabetes, high cholesterol and high white blood cell count $\left(>100,000 / \mathrm{mm}^{3}\right)$. Prior allogeneic haematopoietic cell transplantation was allowed, as long as the patients were not still requiring immunosuppression.

Treatment plan and study design

$A M L$ dose-escalation study. Conducted at three centres in the USA and one in Canada. Dose escalation followed a conventional $3+3$ design. The starting dose of AZD1208 was $120 \mathrm{mg}$ QD orally continuously in each 28-day cycle. In the first cohort, dosing between the first and subsequent patients was staggered with a 7-day interval. Dose-escalation decisions were based on the safety and tolerability data from $\geq 3$ evaluable patients (or six if one of the first three patients experienced a dose-limiting toxicity [DLT]).

Evaluable patients must have received $\geq 75 \%$ of the specified AZD1208 dose, or experienced a DLT, during the first 28-day cycle. AZD1208 could be escalated by up to $100 \%$ in subsequent cohorts until one DLT was observed, after which succeeding doses were escalated by up to $50 \%$.

In the case of a DLT, the cohort was expanded to six patients. If a DLT occurred in $\geq 2$ patients within a cohort, the dose was determined to be a non-tolerated dose (NTD) and dose escalation was stopped.

Grade 3 or 4 toxicities not attributable to the disease or diseaserelated processes under investigation, and occurring before the end of Cycle 1, were considered DLTs. The following were also considered DLTs: QTc prolongation ( $>500 \mathrm{~ms}$; Fridericia's correction) or an increase of $>60 \mathrm{~ms}$ from baseline QTc to a QTc value $>480 \mathrm{~ms}$, confirmed on repeat 48-h electrocardiogram (ECG); Common Terminology Criteria for Adverse Events (CTCAE) grade 3 or 4 vomiting lasting $>24 \mathrm{~h}$, despite suitable antiemetics; grade 5 (death, unless clearly unrelated to therapy [e.g., accidental, due to progressive disease]) or any other toxicity judged to be a DLT by the Safety Review Committee; marrow aplasia continuing for $\geq 42$ days in the absence of leukaemia.

The NTD was identified as the dose at which $\geq 2$ DLTs occurred in a given cohort. The MTD was defined as the highest dose at which $<33 \%$ of six patients experienced a DLT.

Solid tumour dose-escalation study. Conducted at two UK centres and one in Japan. Dose escalation followed a rolling six design. ${ }^{14}$ Patients received a single dose on Day 1 during Cycle 0 , followed by a 3-day washout period, after which multiple dosing was initiated. Cycle 1 was a 21-day period from the first dose of multiple dosing.

The dose decisions for a cohort and definition for an evaluable patient were the same as in the AML dose-escalation study. DLTs were defined as: QTc prolongation ( $>500 \mathrm{~ms}$ ) or an increase of $>60 \mathrm{~ms}$ from baseline QTc to a QTc value $>480 \mathrm{~ms}$, confirmed on repeat 48-h ECG; haematological toxicity ( $\geq$ grade 4 present for $>4$ days [including grade 4 thrombocytopenia, regardless of duration]); anaemia, as defined by haemoglobin $<6.5 \mathrm{~g} / \mathrm{dl} \quad(<4.0 \mathrm{mmol} / \mathrm{l}) ;$ febrile neutropenia (including grade 3 neutropenia and temperatures $>38.5^{\circ} \mathrm{C}$ ); grade 3 thrombocytopenia with grade 3 haemorrhagic events; non-haematological toxicity $\geq$ CTCAE grade 3 , including diarrhoea, nausea or vomiting persisting for $>3$ days despite aggressive management; any other toxicity greater than that at baseline, clinically significant and/or unacceptable, not responding to supportive care and resulting in a disruption of the dosing schedule for $>14$ days.

Administration of AZD1208 began at $120 \mathrm{mg}$ QD, with subsequent dosing levels adjusted based on emerging safety and PD data.

Assessments

Safety. Safety and tolerability were assessed from the time of informed consent until the end of follow-up (defined as 30 and 28 days after study treatment was discontinued for the AML and solid tumour studies, respectively) by evaluation of adverse events (AEs), vital signs, ECGs and laboratory assessments. The CTCAE (version 4.0) was utilised to grade all AE events.

$P K$ sampling. The schedules for collection of blood and urine samples for PK analyses in each cohort in both studies are described in Supplementary Table 1. When necessary for clinical response assessment in the AML study, a bone marrow sample for PK analysis was collected at Cycle 1, Day 28.

In the solid tumour study, blood samples for $4 \beta$-hydroxycholesterol analysis were collected pre-dose at Cycle 0, Day 1; Cycle 1 (Day 8 and 15); and Cycle 2, Day 15.

The concentration of AZD1208 in plasma and urine was determined by Covance, on behalf of Clinical Bioanalysis Alliance at AstraZeneca R\&D, using a bioanalytical method. A volume of $0.05 \mathrm{ml}$ of $\mathrm{K}_{2}$ EDTA human plasma sample was extracted by liquid-liquid extraction using $0.7 \mathrm{ml}$ of methyl tert-butyl ether. Approximately $100 \mu \mathrm{l}$ of the supernatant was transferred, evaporated to dryness and reconstituted with $350 \mu \mathrm{l}$ of 1:1 methanol:water, and a $5 \mu \mathrm{l}$ injection was made to the highperformance liquid chromatography (HPLC) column. HPLC separations were performed on a Phenomenex LUNA C18 $(50 \times 2 \mathrm{~mm}$, $5 \mu \mathrm{m}$ ) column using a mobile phase of $0.1 \%$ formic acid in water (A) and $0.1 \%$ formic acid in acetonitrile (B) at a flow rate of $0.6 \mathrm{ml} / \mathrm{min}$ and a column temperature of $40^{\circ} \mathrm{C}$. AZD1208-D5 was used as the internal standard. Detection was performed in a Sciex API4000 mass spectrometer, in a positive electrospray ionisation mode, using multiple reaction monitoring detection (AZD1208: 380.0->248.1; AZD1208-D5: 385.0->253.1).

\section{PD sampling}

AML dose-escalation study. For AZD1208 PD evaluations, bone marrow aspirates were collected (pretreatment and Cycle 1, Day 1, 2-6 h following administration of AZD1208) and peripheral blood samples were collected (pretreatment on Cycle 1, Day 1; and ontreatment at Cycle 1, Day 1 at 3, 6 and $24 \mathrm{~h}$ post-dose; Cycle 1, Day 14 at pre-dose, 3 and $6 \mathrm{~h}$ post-dose). Mononuclear cells were isolated, and protein lysates prepared, for subsequent analysis of phosphorylated BAD at S112 by MesoScale Discovery ELISA and phosphorylation of 4E-BP1 at S65 by NanoPro immunoassay [unpublished data: McEachern et al. 2018, manuscript in preparation]. Samples were considered evaluable when the baseline 
Table 1. Patient baseline characteristics in the AML and solid tumour dose-escalation studies

\begin{tabular}{|c|c|c|}
\hline & $\begin{array}{l}\text { AML dose-escalation } \\
\text { study } \\
n=32\end{array}$ & $\begin{array}{l}\text { Solid tumour } \\
\text { dose-escalation study } \\
n=35\end{array}$ \\
\hline \multicolumn{3}{|l|}{ Age, years } \\
\hline Median & 65 & 65 \\
\hline Range & $18-89$ & $28-81$ \\
\hline \multicolumn{3}{|l|}{ Sex } \\
\hline Male & $21(65.6)$ & $17(48.6)$ \\
\hline Female & $11(34.4)$ & $18(51.5)$ \\
\hline \multicolumn{3}{|l|}{ Race } \\
\hline White & $26(81.3)$ & $19(54.3)$ \\
\hline Asian & $3(9.4)$ & $16(45.7)$ \\
\hline Other & $3(9.4)$ & 0 \\
\hline \multicolumn{3}{|l|}{ Extent of disease at baseline } \\
\hline Locally advanced & NA & $13(37.1)$ \\
\hline Metastatic & NA & $31(88.6)$ \\
\hline \multicolumn{3}{|l|}{ Disease under study } \\
\hline $\begin{array}{l}\text { Refractory AML } \\
\text { (primary only) }\end{array}$ & $21(65.6)$ & NA \\
\hline First relapse & $3(9.4)$ & NA \\
\hline Second relapse & $4(12.5)$ & NA \\
\hline Third or further relapse & $4(12.5)$ & NA \\
\hline \multicolumn{3}{|l|}{ ECOG PS } \\
\hline 0 & $6(18.8)$ & $16(47.1)$ \\
\hline 1 & $21(65.6)$ & $18(52.9)$ \\
\hline 2 & $5(15.6)$ & 0 \\
\hline \multicolumn{3}{|l|}{ Prior therapy } \\
\hline Surgery & NA & $27(77.1)$ \\
\hline Radiotherapy & $2(6.3)$ & $35(100)$ \\
\hline $\begin{array}{l}\text { Chemotherapy, } n(\%), \\
\text { median }\end{array}$ & $32(100), 4.0$ & 34 (97.1), NA \\
\hline $\begin{array}{l}\text { Immuno-/hormonal } \\
\text { therapy }\end{array}$ & NA & $6(17.1)$ \\
\hline $\begin{array}{l}\text { Other systemic } \\
\text { anticancer therapy }\end{array}$ & $2(6.3)$ & 0 \\
\hline Stem cell transplant & $5(15.6)$ & NA \\
\hline \multicolumn{3}{|l|}{ Molecular mutation status } \\
\hline \multicolumn{3}{|l|}{ FLT3 } \\
\hline Detected & $3(9.4)$ & NA \\
\hline Not detected & $12(37.5)$ & NA \\
\hline Unknown & $17(53.1)$ & NA \\
\hline \multicolumn{3}{|l|}{ NPM1 } \\
\hline Detected & 0 & NA \\
\hline Not detected & $12(37.5)$ & NA \\
\hline Unknown & $20(62.5)$ & NA \\
\hline \multicolumn{3}{|l|}{ Cytogenetics } \\
\hline Normal & $14(43.8)$ & NA \\
\hline $\mathrm{t}(8: 21)$ & $1(3.1)$ & NA \\
\hline Inv 16 or t $(16: 16)$ & $1(3.1)$ & NA \\
\hline $\begin{array}{l}\text { Abnormalities of } 5 \\
\text { and/or } 7\end{array}$ & $7(21.9)$ & NA \\
\hline $\begin{array}{l}\text { Complex (>3 } \\
\text { abnormalities) }\end{array}$ & $8(25.0)$ & NA \\
\hline Other & $14(43.8)$ & NA \\
\hline
\end{tabular}

Data are $n(\%)$ unless otherwise stated. $A M L$ acute myeloid leukaemia, ECOG PS Eastern Oncology Cooperative Group Performance Status, FLT3 FMS-like tyrosine kinase 3, NA not applicable, NPM1 nucleophosmin. aDetails of 'other systemic anticancer prior therapy' are not known biomarker levels were above the background signal (as defined by buffer-only negative control samples) and within the linear range of assay. Additionally, only blood samples containing detectable blasts were considered for analysis.

Isolation of primary leukaemia cells for protein profiling. In the AML study, a protein profiling analysis was carried out on primary blasts obtained from peripheral blood of patients $(n=6)$ before and during therapy with AZD1208. Due to time-sensitive aspects of the analysis, only patients from the MD Anderson Cancer Center (MDACC, Houston, TX, USA) were eligible. Whole blood was collected in heparinised tubes. Leukaemia cells were then isolated using Ficoll-Hypaque (specific gravity, 1.086; Life Technologies, Grand Island, NY, USA) density gradient separation, as previously described. ${ }^{15}$ Cell number and mean cell volume were determined using a Coulter Channelyzer (Coulter Electronics, Hialeah, FL, USA).

Mutation analysis was determined for the AML study patients using the fluorescent multiplex polymerase chain reaction and restriction digestion method, followed by capillary electrophoresis at MDACC.

AML blasts isolated from patients during therapy with AZD1208 were harvested and submitted for reverse phase protein array (RPPA) analysis to evaluate protein level changes across a set of 171 antibodies, ${ }^{16}$ graphed using GraphPad Prism software (GraphPad Software, Inc., San Diego, CA, USA).

Tumour response. The antileukaemic activity in AML was according to the International Working Group (IWG) response criteria for AML. ${ }^{17}$ Response criteria were modified such that an $\mathrm{M}-1$ marrow was defined as $1 \%$ to $\leq 5 \%$ and a partial response as $>5 \%$ to $25 \%{ }^{18}$

In the solid tumour study, responses were evaluated using RECIST v.1.1. Contrast-enhanced CTs of the chest, abdomen, pelvis and neck were performed at screening ( $\leq 28$ days before the start of study treatment), every 6 weeks ( \pm 1 week) up to 12 weeks, and then every 12 weeks ( \pm 1 week) until discontinuation of study treatment or withdrawal of consent, starting from Day 1 of Cycle 1.

Statistical analysis

AEs were summarised by Medical Dictionary for Regulatory Activities (MedDRA) system organ class, MedDRA preferred term and CTCAE grade. Summary statistics of mean, median, standard deviation, minimum, maximum and number of observations were used.

\section{RESULTS}

Baseline data and treatment overview

$A M L$ dose-escalation study. Patient demographics and baseline characteristics were similar in each cohort and are shown in Table 1.

A total of 55 patients were enrolled into the dose-escalation phase of the AML study between 10 February 2012 and 13 May 2014,32 of whom were assigned to treatment. The other 23 patients were screen failures. All patients assigned to treatment received $\geq 1$ dose of AZD1208 at: $120 \mathrm{mg}, n=4 ; 240 \mathrm{mg}, n=6$; $480 \mathrm{mg}, n=6 ; 700 \mathrm{mg}, n=7$; and $900 \mathrm{mg}, n=9$. On Day 28 (Cycle 1 completion), 11 patients were receiving AZD1208 ( $n=2$ in each of the 120,480, 700 and $900 \mathrm{mg}$ dose cohorts; $240 \mathrm{mg}, n=3$ ). Three patients completed Cycle 2 ( $n=1$ in each of the 480, 700 and $900 \mathrm{mg}$ dose cohorts). By Day 84, all patients had discontinued treatment.

Among all dose levels, treatment duration ranged from 4-66 days (median range, 15-27 days). One patient in each of the $240 \mathrm{mg}$ (rash), $480 \mathrm{mg}$ (stomatitis) and $700 \mathrm{mg}$ (febrile neutropenia) dose cohorts, and two patients in the $900 \mathrm{mg}$ cohort (one with hypotension, pyrexia and thrombocytopenia; one with rash), had one dose interruption due to AEs. No patients required dose reduction. All 32 eligible patients eventually discontinued study treatment due to a lack of 
therapeutic response $(n=19,59.4 \%), \operatorname{AEs}(n=8,25.0 \%)$ and patient's decision ( $n=5,15.6 \%)$.

Solid tumour dose-escalation study. Patient demographics and baseline characteristics were similar in each cohort and are shown in Table 1. Overall, 43 patients were enrolled in the solid tumour study between 17 July 2012 and 14 April 2014, 35 of whom were assigned to treatment $(120 \mathrm{mg}, n=3 ; 240 \mathrm{mg}, n=7 ; 360 \mathrm{mg}$, $n=6 ; 540 \mathrm{mg}, n=7 ; 700 \mathrm{mg}, n=6$; and $800 \mathrm{mg}, n=6)$.

Twenty-one (60.0\%) patients completed the Cycle 1, Day 21 DLT evaluation period $(120 \mathrm{mg}, n=3 ; 240 \mathrm{mg}, n=5 ; 360 \mathrm{mg}, n=2$; $540 \mathrm{mg}, n=5 ; 700 \mathrm{mg}, n=3$; and $800 \mathrm{mg}, n=3$ ). Reasons for not completing the DLT evaluation period were progressive disease ( $n=8,22.9 \%)$, patient decision $(n=3,8.6 \%)$ and $\mathrm{AE}(n=3,8.6 \%)$.

The median treatment duration was 41 days, but varied considerably (range, 10-357 days). Mean duration was greater for the lower doses (120-540 mg: 78-91 days) than the higher doses (700 mg and $800 \mathrm{mg}$ : 29-44 days). All 35 (100\%) patients eventually discontinued study treatment due to disease progression $(n=23 ; 65.7 \%)$, patient decision to discontinue $(n=8,22.9 \%)$ or AEs $(n=4,11.4 \%)$.

Safety and tolerability

AML dose-escalation study. AEs: Overall, 31 patients (96.9\%) experienced an AE (Table 2). Gastrointestinal disorders were the most commonly reported AEs $(n=28,87.5 \%)$, most frequently nausea $(n=15,46.9 \%)$ and diarrhoea $(n=14,43.8 \%)$. AEs judged by the investigator to be possibly related to AZD1208 occurred in 22 patients (68.8\%), with the most common being nausea $(n=12$, $37.5 \%)$, diarrhoea $(n=7,21.9 \%)$, vomiting $(n=6,18.8 \%)$ and fatigue ( $n=6,18.8 \%$ ). No clinically significant ECG abnormalities were observed. Seventy-five percent of patients $(n=24)$ experienced a grade $\geq 3 \mathrm{AE}$, with febrile neutropenia $(n=9,28.1 \%)$, hypotension $(n=6,18.8 \%)$ and pneumonia $(n=5,15.6 \%)$ the most commonly reported.

Serious AEs (SAEs) were reported in $71.9 \%(n=23)$ of patients. Febrile neutropenia $(n=8,25.0 \%)$, hypotension, abdominal pain, maculopapular rash and back pain (each $n=2,6.3 \%$ ) were SAEs reported in $>1$ patient. Treatment-related SAEs occurred in five (15.6\%) patients: two in the $700 \mathrm{mg}$ dose cohort (one each of Guillain-Barré syndrome [GBS] and increased blood creatinine) and three in the $900 \mathrm{mg}$ dose cohort (one febrile neutropenia, two rash).

In total, AZD1208 was discontinued in eight patients (25\%), as a result of SAEs and AEs (treatment-related and non-treatmentrelated): $240 \mathrm{mg}, n=1$ (bacteremia [SAE] and peristomal ulcer $[\mathrm{AE}]$ ); $480 \mathrm{mg}, n=2$ (vomiting $[\mathrm{AE}]$ and gingival pain $[\mathrm{AE}]$ ); $700 \mathrm{mg}$, $n=3$ (lung infection, GBS and Escherichia spp. sepsis [SAEs]); and $900 \mathrm{mg}, n=2$ (acute coronary syndrome and rash [SAEs]). These $A E s$ and SAEs were considered treatment-related in four patients: $240 \mathrm{mg}, n=1$ (peristomal ulcer [AE]); $480 \mathrm{mg}, n=1$ (vomiting [AE]); $700 \mathrm{mg}, n=1$ (GBS [SAE]); and $900 \mathrm{mg}, n=1$ (rash [SAE]).

There were nine deaths during the study, all attributed by the investigators to disease progression.

DLTs: DLTs were reported in five patients and occurred between 1-10 days after the start of treatment: $240 \mathrm{mg}, n=1$ (peristomal ulcer); $480 \mathrm{mg}, n=1$ (fatigue); $700 \mathrm{mg}, n=1$ (GBS); $900 \mathrm{mg}, n=2$ (both rash) (Table 3 ). One patient with maculopapular rash was rechallenged with AZD1208 at the same dose with no recurrence of the DLT. The other DLTs of rash and GBS resulted in discontinuation of AZD1208.

During the study, no cohort comprised the minimum of six evaluable patients that was required to define MTD (a total of $3 / 7$ patients completed Cycle 1 at $700 \mathrm{mg}$ ). MTD was not determined as the dose level below the NTD did not contain the six evaluable patients required to define the MTD of AZD1208.
Solid tumour dose-escalation study

AEs: All 35 patients reported $\geq 1 \mathrm{AE}$ (Table 4). Most AEs were gastrointestinal disorders $(n=34,97.1 \%)$, the most common being diarrhoea $(n=29,82.9 \%)$, nausea $(n=26,74.3 \%)$ and vomiting ( $n=19,54.3 \%)$. All 35 patients had an AE considered to be causally related to AZD1208; diarrhoea $(n=24,68.6 \%)$ and nausea $(n=23,65.7 \%)$ were the most common.

$A E s$ of grade $\geq 3$ were observed in 16 patients $(45.7 \%)$. Four patients (11.4\%) discontinued the study due to an $\mathrm{AE}$ : pneumonitis, $n=2$; vomiting, $n=1$; maculopapular rash, $n=1$. Sixteen patients $(45.7 \%)$ required dose interruptions because of AEs: $240 \mathrm{mg}, n=2 ; 360 \mathrm{mg}, n=3 ; 540 \mathrm{mg}, n=6 ; 700 \mathrm{mg}, n=3$; $800 \mathrm{mg}, n=2$. One patient in the $800 \mathrm{mg}$ cohort required dose reduction because of $A E s$.

AEs of CTCAE grade $\geq 3$ that occurred in $\geq 2$ patients included: fatigue $(n=4[11.4 \%])$, gamma-glutamyltransferase (GGT) increase $(n=3[8.6 \%])$, abdominal pain $(n=2[5.7 \%])$ and anaemia $(n=2$ [5.7\%]). CTCAE grade $\geq 3$ AEs causally related to AZD1208 occurred in 10 patients (28.6\%): $240 \mathrm{mg}, n=3$ (anaemia, alanine transaminase increased, GGT increased); $360 \mathrm{mg}, n=1$ (lymphocyte decreased); $540 \mathrm{mg}, n=2$ (vomiting, fatigue, lethargy); $700 \mathrm{mg}$, $n=1$ (nausea, fatigue); $800 \mathrm{mg}, n=3$ (fatigue, hyperglycaemia).

Eight patients (22.9\%) experienced $\geq 1 \mathrm{SAE}$, none of which led to discontinuation of AZD1208. SAEs that occurred in $\geq 2$ patients were dyspnoea $(n=2,5.7 \%)$ and vomiting $(n=2,5.7 \%)$. One patient $(2.9 \%)$ reported three treatment-related SAEs (vomiting, fatigue, general physical health deterioration). One patient (2.9\%) died as a result of an AE (general deterioration of physical health) that was not considered to be AZD1208-related by the investigator.

No clinically significant treatment-related changes in haematology, clinical chemistry, vital signs, ECG or physical findings were detected in any patient.

Four patients experienced DLTs: grade 3 fatigue (800 $\mathrm{mg}, n=2)$, grade 3 GGT increase $(240 \mathrm{mg}, n=1)$ and grade 3 vomiting (540 mg, $n=1$ ) (Table 3).

During the study, the $800 \mathrm{mg}$ dose was not expanded to the six evaluable patients required to define MTD. As no dosing cohort met these criteria, the MTD of AZD1208 could not be determined.

PK

AML dose-escalation study. The absorption of AZD1208 after a single dose was rapid, with median time to maximum plasma concentration $\left(\mathrm{T}_{\max }\right)$ of $\sim 3 \mathrm{~h}$ and concentrations remaining high until $24 \mathrm{~h}$ (Supplementary Table 2 and Supplementary Figure 1A). A statistical power analysis demonstrated that following a single dose, the maximum AZD1208 plasma concentration $\left(C_{\max }\right)$ (Supplementary Figure 2) and area under plasma concentration-time curve $\left(A \cup C_{0-t}\right)$ (Supplementary Figure 3) generally increased in proportion to the administered dose across cohorts (range, 120-900 mg).

Renal clearance was low across all cohorts, with $<1 \%$ of the administered dose eliminated unchanged in urine within $24 \mathrm{~h}$. Absorption of AZD1208 was likewise rapid after multiple doses, but highly variable, with up to 10-fold differences in AZD1208 concentrations between individuals even within the same cohort (Supplementary Table 2 and Supplementary Figure 1B).

Across the $120-900 \mathrm{mg}$ doses, $25 \%(4 / 16)$ of patients had significantly lower exposure at steady state compared with the first dose of AZD1208, and the percentage of patients with a lower accumulation ratio increased with increasing dose $(0 \%$ at $240 \mathrm{mg}$ to $67 \%$ at $900 \mathrm{mg}$ ). However, $44 \%$ (7/16) of patients did show a marked accumulation ( $>3$-fold) across the doses. The number of patients with higher accumulation decreased with increasing doses, and exposure was highly variable, with one patient at $480 \mathrm{mg}$ having an accumulation ratio of 3.6 and another a ratio of 0.36 .

In general, both $A U C$ over dosing interval $\left(A \cup C_{\text {tau }}\right)$ and $C_{\max }$ decreased with increasing doses. Power and ANOVA models 


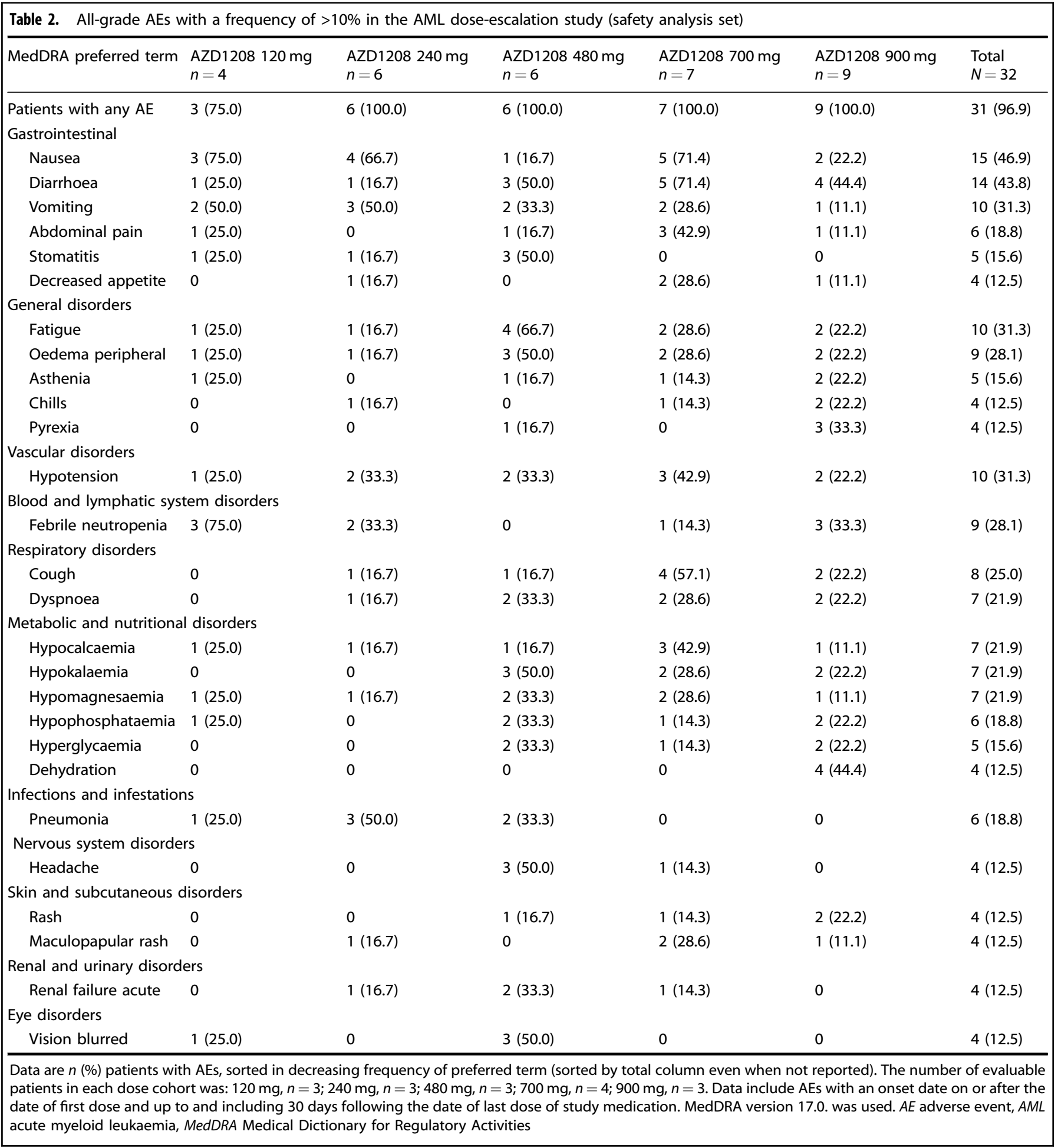

revealed that whereas $C_{\max }$ and $\mathrm{AUC}_{0-24}$ were dose proportional on Cycle 1, Day 1, both $C_{\max }$ and $\mathrm{AUC}_{0-24}$ were less than dose proportional following multiple doses of AZD1208.

Solid tumour dose-escalation study. After a single dose, the systemic exposure of AZD1208 (AUC and $C_{\max }$ ) was variable but largely proportional with doses up to $700 \mathrm{mg}$, and less than proportional from $700-800 \mathrm{mg}$ (Fig. 1a and Supplementary Table 3). After multiple dosing, exposure at all doses was similar, with no increase in exposure with increasing doses (Fig. 1b and
Supplementary Table 3). Individual and geometric mean values for $\mathrm{C}_{\max }$ and AUC are presented in Supplementary Figures 2 and 3, respectively.

The mean half-life following a single dose was determined to be $37.2 \mathrm{~h} \mathrm{(min/max:} 18.9 \mathrm{~h} / 103 \mathrm{~h}$ ). The elimination half-life after multiple dosing could not be accurately determined.

Absorption following single dosing was moderate and variable, with median $\mathrm{T}_{\max }$ achieved by $\sim 5 \mathrm{~h}$ (range, 1.5-25 h); distribution was moderate, and clearance low. In comparison, after multiple dosing, the absorption was moderate, with median $\mathrm{T}_{\max }$ of $\sim 4 \mathrm{~h}$ 
(range, 1.5-6 h), and clearance increased with increasing dose.

After a single dose, $\sim 1 \%$ of the AZD1208 dose was eliminated in urine over $72 \mathrm{~h}$. After multiple dosing, on Day $150.5 \%$ of the AZD1208 dose was observed in urine. Therefore, renal clearance was negligible.

The activity of CYP3A4 was assessed by analysing 4- $\beta$-hydroxycholesterol levels in samples collected from five patients at AZD1208 doses of 700 or $800 \mathrm{mg}$. At both doses, the levels of 4 - $\beta$-hydroxycholesterol increased $\sim 4$-fold in all patients on Day 15, compared with Day 1 (Supplementary Table 4), indicating that CYP3A4 activity was induced by AZD1208.

PD

Reductions in pBAD S112 were observed in 7/17 patients with evaluable bone marrow samples in the AML study (Fig. 2a). Reductions in p4E-BP1 S65 were also observed in 3/11 patients with evaluable bone marrow samples (Fig. 2b). However, the small number of patients and variability in PD response in these data must be noted.

Reductions of pBAD $\mathrm{S} 112$ in peripheral blood were more frequently observed, with $9 / 10$ patients across all dose levels demonstrating $\geq 50 \%$ reduction from baseline (Fig. 2a). Decreases in p4E-BP1 S65 were seen in the peripheral blood samples from 4/7 patients, with the greatest reductions observed at the highest dose level of $900 \mathrm{mg}$ (Fig. 2b). No correlation between dose, exposure and biomarker effect was identified in these samples.

AML study-RPPA protein profiling in AML blasts

RPPA analysis of 171 proteins was performed on samples from six patients (Supplementary Figure 1A). Based on the PD analyses using MesoScale (Meso Scale Diagnostics, LLC, MD, USA) and NanoPro (ProteinSimple, CA, USA), and on previous preclinical investigations in AML cell lines, ${ }^{19}$ five phosphoproteins were selected for analysis. Consistent with Fig. 2, there was heterogeneity in the protein level changes of phosphorylated 4E-BP1 S65 and BAD S112, measured using RPPA analysis (Supplementary Figures $4 \mathrm{~A}$ and $4 \mathrm{~B}$ ). Reductions were seen in $4 \mathrm{E}-\mathrm{BP} 1 \mathrm{~S} 65$ in three patients (Supplementary Figure 4A) and in BAD S112 in four patients (Supplementary Figure 4B). However, the small number of patients and variability in response in these data must be noted. Similarly, there was variability in the effects on phosphorylated 4E-BP1 T37/46, PRAS40 T246 and mTOR S2448 levels following AZD1208 treatment (Supplementary Figures 5A-5D).

Efficacy

$A M L$ dose-escalation study. There were no clinical responses according to IWG criteria (Supplementary Table 5). A reduction in circulating blasts occurred in several patients in the 120, 240 and $480 \mathrm{mg}$ dose cohorts. Only five patients who displayed decreases in circulating blasts had evaluable biomarker samples, three of whom showed a robust decrease in phosphoprotein levels. However, several patients had reduced phosphoprotein levels without a reduction in blasts. Resistant disease was the most common treatment failure reason.

Solid tumour dose-escalation study. The best objective response, as assessed by RECIST, was stable disease for $\geq 6$ weeks $(n=13)$ and progression $(n=14)$, with five patients non-evaluable for response assessment (Supplementary Table 5). Patients were not evaluable because they had stable disease response for $<6$ weeks $(n=3)$ or incomplete post-baseline assessments $(n=2)$. The objective response rate for the study was $0 \%$. At Week 12 , only five patients were evaluable for tumour response assessments: four patients had stable disease (120 mg, $n=1 ; 240 \mathrm{mg}, n=2$ and $540 \mathrm{mg}, n=1)$; one had progressive disease $(240 \mathrm{mg})$. Of note, one patient who received treatment in the solid tumour doseescalation study had prostate cancer, and experienced a considerable reduction in prostate-specific antigen (PSA) levels. 


\begin{tabular}{|c|c|c|c|c|c|c|c|}
\hline \multicolumn{8}{|l|}{ Gastrointestinal disorders } \\
\hline Diarrhoea & $2(66.7)$ & $5(71.4)$ & $5(83.3)$ & $6(85.7)$ & $5(83.3)$ & $6(100.0)$ & $29(82.9)$ \\
\hline Nausea & $1(33.3)$ & $6(85.7)$ & $3(50.0)$ & $7(100.0)$ & $5(83.3)$ & $4(66.7)$ & $26(74.3)$ \\
\hline Abdominal pain & $0(0.0)$ & $3(42.9)$ & $2(33.3)$ & $2(28.6)$ & $1(16.7)$ & $0(0.0)$ & $8(22.9)$ \\
\hline Constipation & $0(0.0)$ & $0(0.0)$ & $3(50.0)$ & $0(0.0)$ & $2(33.3)$ & $0(0.0)$ & $5(14.3)$ \\
\hline \multicolumn{8}{|c|}{ Blood and lymphatic system disorders } \\
\hline Anaemia & $3(100.0)$ & $4(57.1)$ & $2(33.3)$ & $4(57.1)$ & $3(50.0)$ & $2(33.3)$ & $18(51.4)$ \\
\hline Thrombocytopenia & $0(0.0)$ & $0(0.0)$ & $2(33.3)$ & $1(14.3)$ & $1(16.7)$ & $1(16.7)$ & $5(14.3)$ \\
\hline Decreased appetite & $1(33.3)$ & $3(42.9)$ & $2(33.3)$ & $4(57.1)$ & $2(33.3)$ & $3(50.0)$ & 15 (42.9) \\
\hline Hypoalbuminaemia & $1(33.3)$ & $2(28.6)$ & $2(33.3)$ & $1(14.3)$ & $3(50.0)$ & $0(0.0)$ & $9(25.7)$ \\
\hline Hyperglycaemia & $1(33.3)$ & $0(0.0)$ & $1(16.7)$ & $4(57.1)$ & $0(0.0)$ & $2(33.3)$ & $8(22.9)$ \\
\hline Hyponatraemia & $0(0.0)$ & $0(0.0)$ & $1(16.7)$ & $3(42.9)$ & 1 (16.7) & $0(0.0)$ & $5(14.3)$ \\
\hline Hypokalaemia & $0(0.0)$ & $0(0.0)$ & $0(0.0)$ & $2(28.6)$ & $1(16.7)$ & $1(16.7)$ & $4(11.4)$ \\
\hline \multicolumn{8}{|l|}{ Investigations ${ }^{\mathrm{a}}$} \\
\hline Platelet count decreased & $2(66.7)$ & $2(28.6)$ & $1(16.7)$ & $2(28.6)$ & $1(16.7)$ & $1(16.7)$ & $9(25.7)$ \\
\hline $\begin{array}{l}\text { White blood cell count } \\
\text { decreased }\end{array}$ & $2(66.7)$ & $3(42.9)$ & $1(16.7)$ & $2(28.6)$ & $1(16.7)$ & $0(0.0)$ & $9(25.7)$ \\
\hline GGT increased & $0(0.0)$ & $2(28.6)$ & $1(16.7)$ & $2(28.6)$ & $0(0.0)$ & $1(16.7)$ & $6(17.1)$ \\
\hline $\begin{array}{l}\text { Musculoskeletal chest } \\
\text { pain }\end{array}$ & $0(0.0)$ & $0(0.0)$ & $2(33.3)$ & $1(14.3)$ & $1(16.7)$ & $0(0.0)$ & $4(11.4)$ \\
\hline
\end{tabular}

\section{DISCUSSION}

In the first-in-human, dose-escalation study in patients with heavily pretreated AML, AZD1208 was generally tolerated up to doses of $700 \mathrm{mg}$, but not tolerated at the highest $900 \mathrm{mg}$ dose. PK data from this study suggest that AZD1208 absorption profiles for patients who experienced DLTs were in the same range as those of patients who did not have a DLT. It is unlikely that dose interruptions contributed to any variability in repeat dosing PK as the majority of the PK sampling timepoints occurred early in the study (first 15 days of treatment in Cycle 1). In the first 15 days of treatment there were minimal to no dose interruptions, and those patients who had dose interruptions were not included in the PK statistical analysis and hence did not contribute to the variability. In the solid tumour dose-escalation study, AZD1208 was tolerated as monotherapy at doses up to $700 \mathrm{mg}$ QD, but not tolerated at $800 \mathrm{mg}$ QD. The MTD was not confirmed in either study. It is possible that the dose below $900 \mathrm{mg}$ was the MTD; however, as we did not enrol the six patients required by the protocol, this cannot be confirmed.

Pan-PIM inhibition with AZD1208 appeared to be generally tolerated in both studies, with the most common AEs affecting the gastrointestinal tract. The patient in the AML study with GBS had a history of $E$. coli bacteremia a few weeks before starting treatment with AZD1208, and concurrent diseases including polyarthritis and gout. Previous anticancer therapies included ruxolitinib, vidaza, vosaroxin, decitabine, fludarabine and cytarabine. In light of this history, it was concluded that these factors may have contributed to the development of GBS.

Another pan-PIM kinase inhibitor, LGH447, is currently in clinical development and shows a manageable safety profile (ClinicalTrials.gov, NCT01456689). ${ }^{20}$ Among the 54 patients who received LGH447 as monotherapy, there were eight DLTs (thrombocytopenia, $n=4$; fatigue, $n=2$; hypophosphatemia, $n=1$; vasovagal syncope, $n=1$ ), but most AEs were grade 

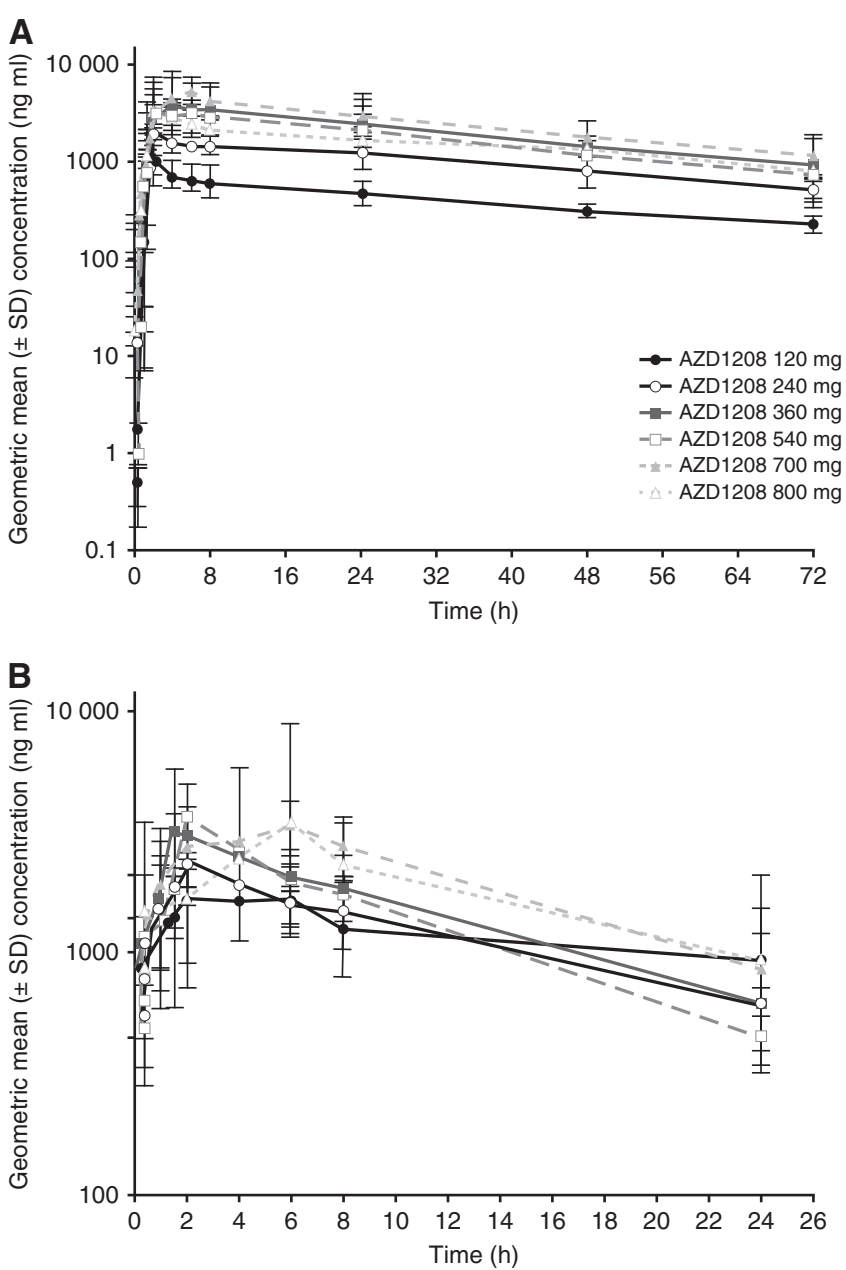

Fig. 1 Solid tumour dose-escalation study geometric mean plasma concentration (semi-log scales \pm SD) of AZD1208 vs time by a single dose for Cycle 0, Day 1, and b multiple doses for Cycle 1, Day 15. $S D$, standard deviation

1 or $2 .^{20}$ While pan-inhibition of PIM kinases with AZD1208 and LGH447 appears to be tolerable, development of the pan-PIM kinase inhibitor SGI-1776 in refractory prostate cancer and relapsed/refractory non-Hodgkin lymphoma was discontinued because of QTc prolongation (ClinicalTrials.gov, NCT00848601).

The lack of clinical responses in our AML and solid tumour doseescalation studies suggests that targeting the PIM pathway with monotherapy may be insufficient to impact refractory $A M L$ or advanced solid malignancies. However, PD analysis of AZD1208 activity showed that, in a subset of patients, AZD1208 treatment resulted in a reduction in the phosphorylation of PIM targets, providing evidence for the biological activity of AZD1208 in patients with refractory AML.

Pan-PIM inhibition has demonstrated therapeutic efficacy in a phase I study of LGH447 in 54 patients with relapsed/refractory multiple myeloma (RRMM). ${ }^{20}$ The overall response rate was $10.4 \%$, with a minor response or greater in $20.8 \%$ and stable disease or greater in $68.8 \%{ }^{20}$ Therefore, identifying the tumour types that may be more sensitive to PIM inhibition will be important for future PIM inhibitor trials. For instance, PIM overexpression has been strongly associated with prostate cancer; ${ }^{21,22}$ indeed, one patient who received treatment in the solid tumour doseescalation study had prostate cancer, and experienced a considerable reduction in PSA levels. In MYC-driven prostate cancer models, AZD1208 significantly decreased tumour growth, an effect that was accompanied by decreased cellular proliferation
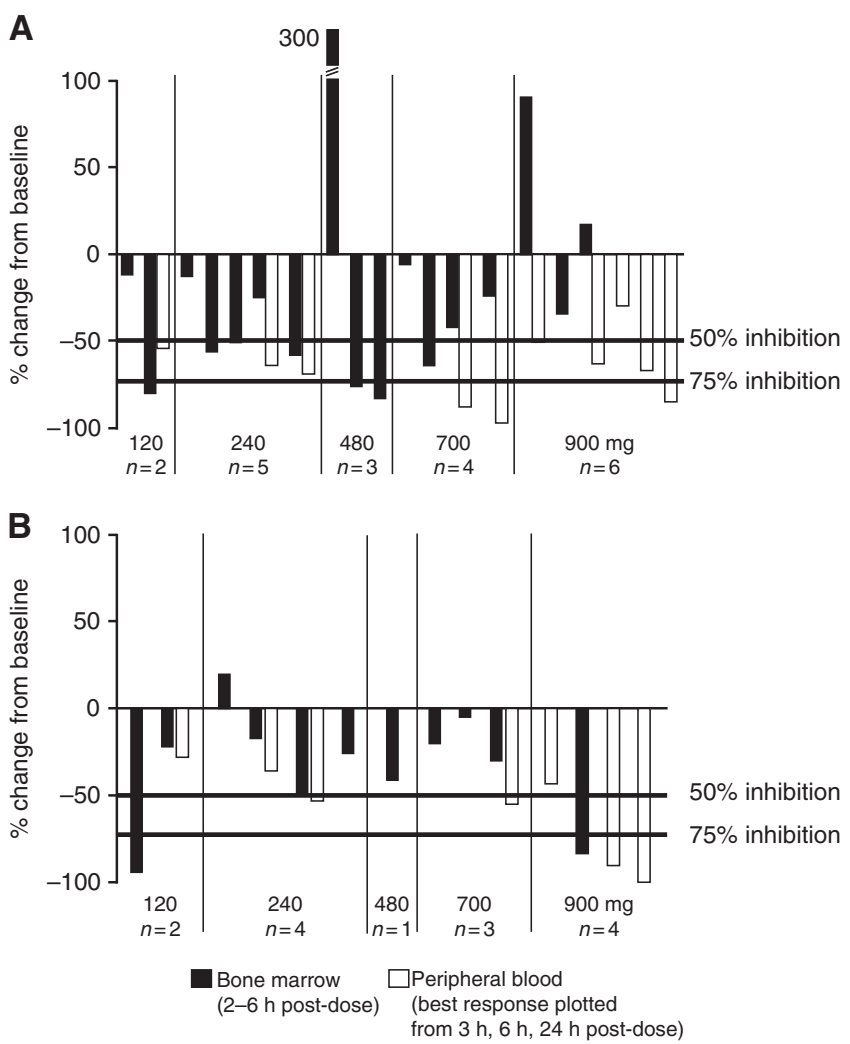

Fig. 2 PD response to AZD1208 treatment in the AML doseescalation study. The percentage of change from baseline in the reduction of (a) pBAD S112, and (b) p4E-BP1 S65 in bone marrow and peripheral blood samples collected following a single dose of AZD1208 is shown. Only the maximal PD response measured on Day 1 for peripheral blood samples is shown. Each column represents an individual patient, with adjacent columns corresponding with matched marrow and blood samples. $A M L$, acute myeloid leukaemia; $P D$, pharmacodynamics

and increased rates of apoptosis. AZD1208 treatment also sensitised the prostate tumours to radiation. ${ }^{13}$

PIM kinase inhibitors may have the potential to be used in combination with other therapies. For example, a phase $\mathrm{lb} / \mathrm{II}$ trial of LGH447 in combination with the PI3K inhibitor, BYL719, is underway in patients with RRMM (ClinicalTrials.gov, NCT02144038). In preclinical tumour models, PIM kinase inhibitors also have the ability to sensitise cancer cells to radiotherapy and chemotherapy. ${ }^{23,24}$ For example, in a mouse xenograft model of non-small-cell lung cancer, PIM inhibition sensitised cancer cells to radiation ${ }^{23}$ and PIM antagonism in prostate cancer cells sensitised the cells to the chemotherapeutic gemcitabine. ${ }^{24}$ Thus, PIM kinase inhibitors may be important in contexts where PIM kinases are acting with other therapeutic targets to drive oncogenic progression.

Preclinical studies of AML suggest that mTOR pathway signalling and suppression of protein translation may play a part in the mechanism of action of AZD1208. ${ }^{19}$ Consistent with these studies, significant reductions in 4EBP1 S65 were seen in a subset of patients while more modest decreases in 4E-BP1 T37/46 and mTOR S2448 were also noted. Given the lack of clinical efficacy in the AML trial, it is not clear to what extent effects on protein translation contribute to PIM kinase activity in AML.

Based on the half-life of AZD1208 after a single dose, it was predicted that multiple dosing would lead to accumulation of AZD1208. However, exposure decreased with increasing doses and with duration of dosing, indicating a possible change in 
clearance. Increased levels of 4- $\beta$-hydroxycholesterol following multiple dosing of AZD1208 confirmed that the increase in clearance was due to induction of CYP3A4 enzymatic activity.

In conclusion, AZD1208 was generally tolerated in patients with heavily pretreated AML and advanced solid malignancies in two dose-escalation studies. AZD1208 increased CYP3A4 activity after multiple dosing, resulting in increased drug clearance. There was no clear evidence of antitumour activity with AZD1208 monotherapy, and the MTD was not established. Considering the challenges in managing this potent increase in CYP3A4 activity, in addition to the lack of observed responses in the clinical setting, the development of AZD1208 was terminated. Still, PIM kinase inhibition may be a relevant anticancer strategy, potentially in combination with other agents.

\section{ACKNOWLEDGEMENTS}

Medical writing support, under the direction of the authors, was provided by Thomas Owens, PhD, of CMC CONNECT, a division of Complete Medical Communications Ltd, Macclesfield, UK, funded by AstraZeneca, Cambridge, UK, in accordance with Good Publication Practice (GPP3) guidelines. This study was funded by AstraZeneca.

\section{AUTHOR CONTRIBUTIONS}

Conception and design: J.C., E.D., D.J.D.; Acquisition of data (provided animals, acquired and managed patients, provided facilities, etc.): J.C., D.J.D., D.L., G.L.U., L.S.C., V.G., E.D.; Analysis and interpretation of data (e.g., statistical analysis, biostatistics, computational analysis): J.C., K.T., D.J.D., Jde.B., D.L., M.M., G.L.U., H.K., L.S.C., V.G., R.G., K.K., K.M., K.V., J.E.P., E.D.; Writing, review and/or revision of the manuscript: J.C., K.T., D.J.D., Jde.B., D.L., M.M., G.L.U., H.K., L.S.C., V.G., R.G., K.K., K.M., K.V., J.E.P., E.D.

\section{ADDITIONAL INFORMATION}

Supplementary information is available for this paper at https://doi.org/10.1038/ s41416-018-0082-1.

Competing interests: J.C. has received research support from, and served as a consultant for, AstraZeneca. D.J.D. has received honoraria from AstraZeneca for participation in advisory boards. Jde.B. has served on AstraZeneca advisory boards. L.S.C. has received honoraria from AstraZeneca for participation in advisory boards. V.G. has received research support and honoraria from AstraZeneca for participation in advisory boards. R.G., K.V. and J.E.P. are employees of, and hold stock in, AstraZeneca. K.K. and K.M. are former employees of AstraZeneca. E.D. is currently employed by AstraZeneca but for the duration of the study was affiliated to The Christie NHS Foundation Trust and The University of Manchester. E.D.'s former institution-The Christie NHS Foundation Trust-has received commercial income from AstraZeneca. The clinical research was supported by Experimental Cancer Medicine grant award C1467/A15578. K.T., D.L., M.M., G.L.U. and H.K. have no conflicts of interest or disclosures to declare.

Ethics approval and consent to participate: Prior to participation in either study, all patients signed an informed consent document approved by the Institutional Review Board. For the solid tumour study, ethical approval was given by the Institutional Review Boards at the three study sites: National Cancer Center Hospital (Tokyo, Japan), the Christie Hospital NHS Trust (Manchester, UK) and the Institute of Cancer Research and Royal Marsden NHS Foundation (Surrey, UK). For the AML study, ethical approval was given by the Institutional Review Boards at the four study sites: MD Anderson Cancer Center (Houston, USA), Dana-Farber Cancer Institute (Boston, USA), Princess Margaret Hospital (Toronto, Canada), and Washington University School of Medicine (St Louis, USA). Both studies were conducted according to the Declaration of Helsinki.

Funding: This study was funded by AstraZeneca. This work was also supported in part by Cancer Center Support Grant (CCSG) P30 CA016672 and by the Translational Research Program of the Leukemia and Lymphoma Society of America (R6011-14).

\section{REFERENCES}

1. Aho, T. L. et al. Pim-1 kinase promotes inactivation of the pro-apoptotic Bad protein by phosphorylating it on the Ser112 gatekeeper site. FEBS Lett. 571, 43-49 (2004)

2. Yan, $B$. et al. The PIM-2 kinase phosphorylates BAD on serine 112 and reverses BAD-induced cell death. J. Biol. Chem. 278, 45358-45367 (2003).
3. Wang, Z. et al. Phosphorylation of the cell cycle inhibitor p21Cip1/WAF1 by Pim-1 kinase. Biochim Biophys. Acta 1593, 45-55 (2002).

4. Winn, L. M., Lei, W. \& Ness, S. A. Pim-1 phosphorylates the DNA binding domain of c-Myb. Cell Cycle 2, 258-262 (2003).

5. Cuypers, H. T. et al. Murine leukemia virus-induced T-cell lymphomagenesis: integration of proviruses in a distinct chromosomal region. Cell 37, 141-150 (1984).

6. van Lohuizen, $M$. et al. Predisposition to lymphomagenesis in pim-1 transgenic mice: cooperation with c-myc and $\mathrm{N}$-myc in murine leukemia virus-induced tumors. Cell 56, 673-682 (1989).

7. Amson, R. et al. The human protooncogene product p33pim is expressed during fetal hematopoiesis and in diverse leukemias. Proc. Natl Acad. Sci. USA 86, 8857-8861 (1989).

8. Asano, J. et al. The serine/threonine kinase Pim-2 is a novel anti-apoptotic mediator in myeloma cells. Leukemia 25, 1182-1188 (2011).

9. Cohen, A. M. et al. Increased expression of the hPim-2 gene in human chronic lymphocytic leukemia and non-Hodgkin lymphoma. Leuk. Lymphoma 45, 951-955 (2004).

10. Poulsen, C. B. et al. Microarray-based classification of diffuse large B-cell lymphoma. Eur. J. Haematol. 74, 453-465 (2005).

11. Dakin, L. A. et al. Discovery of novel benzylidene-1,3-thiazolidine-2,4-diones as potent and selective inhibitors of the PIM-1, PIM-2, and PIM-3 protein kinases. Bioorg. Med Chem. Lett. 22, 4599-4604 (2012).

12. Keeton, E. K. et al. AZD1208, a potent and selective pan-Pim kinase inhibitor, demonstrates efficacy in preclinical models of acute myeloid leukemia. Blood 123, 905-913 (2014).

13. Kirschner, A. N. et al. PIM kinase inhibitor AZD1208 for treatment of MYC-driven prostate cancer. J. Natl. Cancer Inst. 107, dju407 (2014).

14. Skolnik, J. M., Barrett, J. S., Jayaraman, B., Patel, D. \& Adamson, P. C. Shortening the timeline of pediatric phase I trials: the rolling six design. J. Clin. Oncol. 26, 190-195 (2008).

15. Chen, L. S., Keating, M. J. \& Gandhi, V. Blood collection methods affect cellular protein integrity: implications for clinical trial biomarkers and ZAP-70 in CLL. Blood 124, 1192-1195 (2014).

16. The University of Texas MD Anderson Cancer Center. Functional Proteomics RPPA Core Facility. www.mdanderson.org/research/research-resources/core-facilities/ functional-proteomics-rppa-core.html (2017).

17. Cheson, B. D. et al. Revised recommendations of the international working group for diagnosis, standardization of response criteria, treatment outcomes, and reporting standards for therapeutic trials in acute myeloid leukemia. J. Clin. Oncol. 21, 4642-4649 (2003).

18. Kantarjian, $\mathrm{H}$. et al. Phase 2 clinical and pharmacologic study of clofarabine in patients with refractory or relapsed acute leukemia. Blood 102, 2379-2386 (2003).

19. Chen, L. S., Yang, J. Y., Liang, H., Cortes, J. E. \& Gandhi, V. Protein profiling identifies mTOR pathway modulation and cytostatic effects of Pim kinase inhibitor, AZD1208, in acute myeloid leukemia. Leuk. Lymphoma 57, 2863-2873 (2016).

20. Raab MS, et al. Phase 1 study update of the novel Pan-Pim kinase inhibitor LGH447 in patients with relapsed/refractory multiple myeloma. Blood. 124, abstr. 301 (2014).

21. Nawijn, M. C., Alendar, A. \& Berns, A. For better or for worse: the role of Pim oncogenes in tumorigenesis. Nat. Rev. Cancer 11, 23-34 (2011).

22. Shah, $\mathrm{N}$. et al. Potential roles for the PIM1 kinase in human cancer-a molecular and therapeutic appraisal. Eur. J. Cancer 44, 2144-2151 (2008).

23. Kim, W. et al. PIM1 kinase inhibitors induce radiosensitization in non-small cell lung cancer cells. Pharmacol. Res 70, 90-101 (2013).

24. Xu, D. et al. Inhibition of oncogenic Pim-3 kinase modulates transformed growth and chemosensitizes pancreatic cancer cells to gemcitabine. Cancer Biol. Ther. 14, 492-501 (2013).

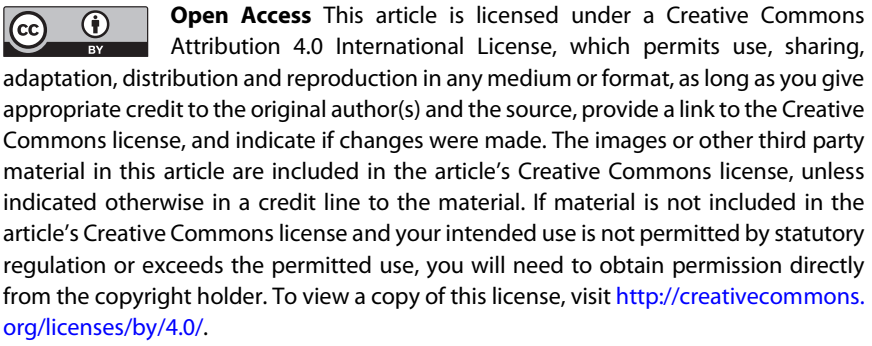

(c) The Author(s) 2018 